\title{
Quelques réflexions sur la conception japonaise de l'aménagement des pêches
}

\author{
par Denis BAILLY
}

Département Stratégies de Développement et d'Aménagement, IFREMER, 66, avenue d'léna, 75116 Paris.

\begin{abstract}
RESUME
Débattre d'aménagement et de sociétés littorales implique nécessairement de parler de pêche cotière et de culuures marines. Face aux limites et contraintes économiques, sociales et biologiques révélées et/ou imposées aux pêcheries par les conditions propres de leur développement ou par le développement industriel, touristique, urbain,... la nécessité de leur aménagement se fait de plus en plus fortement sentir.

Le débat sur la nature à la fois liée et contradictoire de l'aménagenent des pêches et du caractère public des ressources et de l'espace maritime cotter n'a occupé que très peu de place dans l'histoire des pêches japonaises. Au moment où, en France ce débat semble laisser place à la discussion des modalités de la gestion et de l'aménagement des pêcheries, il est intéressant de jeter un regard sur les conceptions qui prévalent dans l'aménagement des pêches au Japon.

En présentant les principaux volets et leur évolution hislorique on examinera en quoi une conception qui met au centre de ses préoccupations les aspects économiques et sociaux diffère dans ses implications d'une conception qui assigne une fonction d'aménagement à l'objectif de préservation de la ressource. Il en résulte une histoire, des pratiques, une hiérarchisation des objectifs et des choix, qui sont uniques au monde et dont nous avons peutêtre des leçons à tirer.
\end{abstract}

Mots-clés : Pêche côtière. Cultures marines. Aménagement. Tapon.

Key-words : Coastal Fisheries. Marine Cultures. Management. Japan. 


\section{ABSTRACT}

Coastal fisheries and marine cultures developed under strong biological, economical and social constraints. Among others are limitation of space and resources, limited avalibility of capilal, hardship of work... Industrial, urbanistic and touristic development add to these constraints in coastal areas. Coherent scheme for coastal fisheries management, capture and culure, is of an urgent need.

In renewable resources and environnement econonics, depletion and pollution resulting from common ownership have been the cone of the theory about fisheries management. The chronology of japanese coastal fisheries management system shows a different approach. In law and decision making, social and economic consequences of conflictual uses and of their regulation are given a large place.

In such matter there cannot be any model. Historical, past and present, circumstances are not similar. However the discussion of advantages and limits of the japanese system may still be useful.

Chaque jour les médias japonais se font l'écho des conflits suscités par l'utilisation de l'espace et des ressources du littoral. Les pollutions industrielles et urbaines, le développement de l'aquaculture et la place des produits marins dans l'alimentation japonaise sont des images que nous connaissons tous. L'harmonisation des conditions de vie des sociétés littorales et la gestion de l'espace côtier sont un souci quotidien pour plus de 40 milions de japonais qui vivent dans des communes ou agglomérations littorales. Villes et industries s'étalent le long des côtes avec une concentration unique au monde. D'autres chiffres donnent l'importance de l'accès au littoral. $90 \%$ des matières premières et de l'énergie, ainsi que la majeure partie des exportations, transitent par la mer. Près de $50 \%$ des protéines animales consonmées sont d'origine marine, 2800 ports abritent les activités de 450000 aquaculteırs et pêcheurs côtiers ct hauturiers qui font vivre directement 1300000 personnes. La carte des principaux ports de débarquement et concentrations urbaines (fig. 1) donne une idée de l'ampleur des problèmes.

Lcs pêches côtières et hauturières se sont développées dans des eaux exceptionnellement riches et dans un espace et un environnement littoral très disputés. Jouissant par le passé d'un quasi monopole de l'exploitation de l'interface terre-mer elles ont dû relever les défis du développement industriel et urbain. Leur histoire a produit un schéma d'aménagement original et des activités qui globalement n'ont cessé de se montrer plus performantes et mieux organisées. Ceci ne s'est pas fait sans de nombreuses difficultés. L'avenir de cet aménagement est aujourd'hui menacé par le développement de nouvelles activités (tourisme, centrales thermiques,...) et par la baisse du revenu des pêcheurs. Nous tenterons ici de dégager ce qui fait l'originalité du système japonais d'aménagement des pêches dans sa conception et ses principes. Pour cela on en présentera tout d'abord lc schéma général. Quelques rappels historiques permettront de replacer ce schéma dans le contexte économique, politique et social du Japon. Enfin certains aspects mettant en lumière ses limites seront discutés. 
- Principaux ports de débarquement
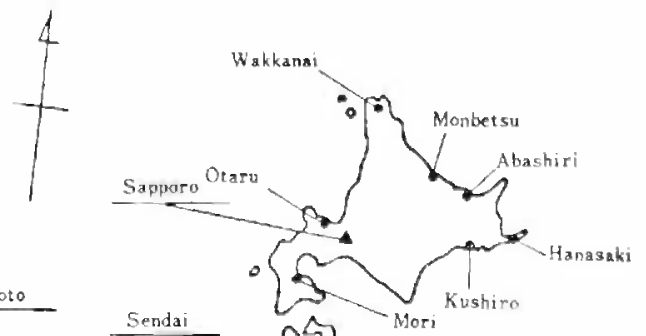

- 10 plus grandes concentrations urbaines

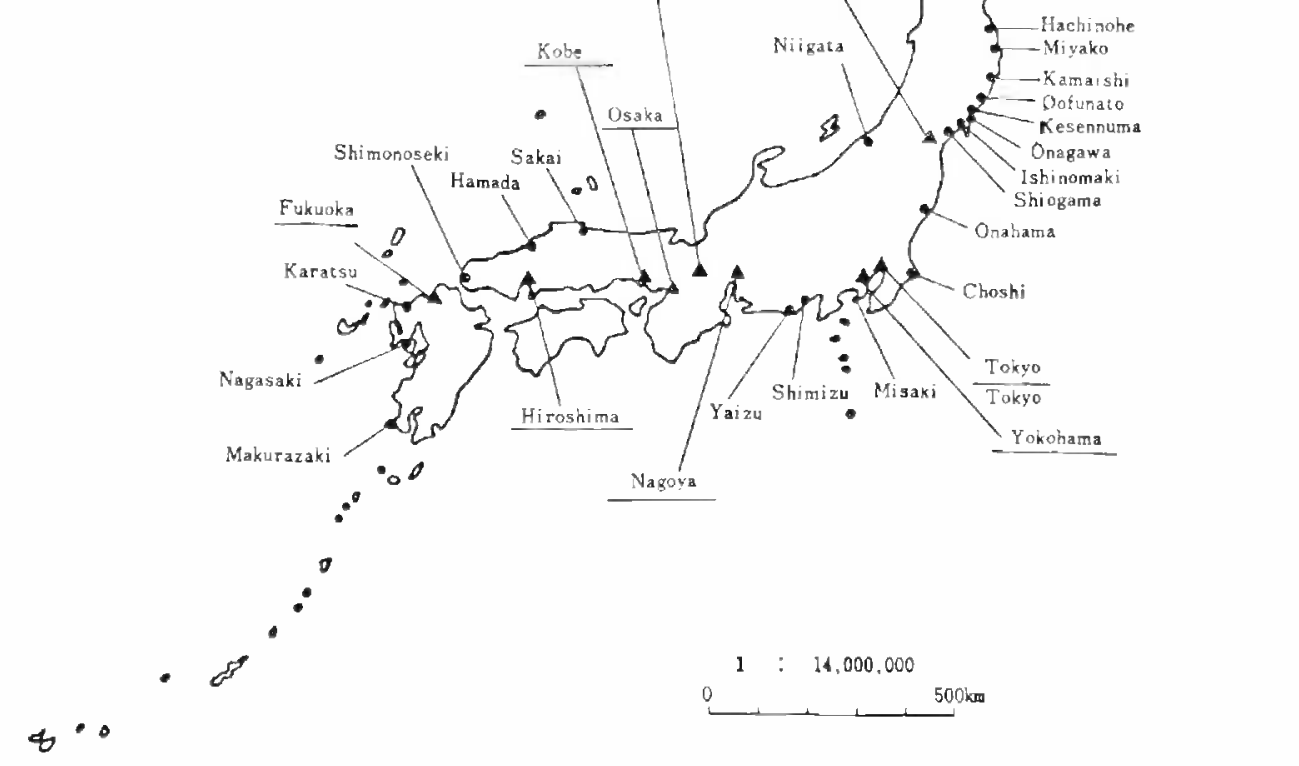

FIG. 1. - Principaux ports de débarquement ef les dix plus grandes concentrations urbaines.

La gestion des pêcheries, et plus largement l'aménagement de l'utilisation des espaces littoraux, soulèvent de nombreux débats en France et en Europe. Dans cette période où les réponses japonaises aux difficultés des sociétés industrielles sont trop souvent idéalisées nous essaierons de faire la part des choses entre la réalité, les fondements de l'aménagement, leur efficience et leurs limites dans le contexte japonais.

\section{I. - LE SYSTEME D'AMENAGEMENT DES PECHES JAPONAIS.}

Le Japon occupe le premier rang mondial en matiere de productions marines. En 1985 les apports étaient de 12 millions de tonnes. La part de la grande pêche était de $17 \%$, celle de la pêche hauturière de $55 \%$, avec $19 \%$ pour la petite pêche cotière. Les $9 \%$ restants ont été produits par l'aquaculture. En valeur, la contribution des pêches côtières et hauturières est encore plus importante. La pêche hauturière est majoritairement artisanale. La législation établit un schéma d'aménagement de la 


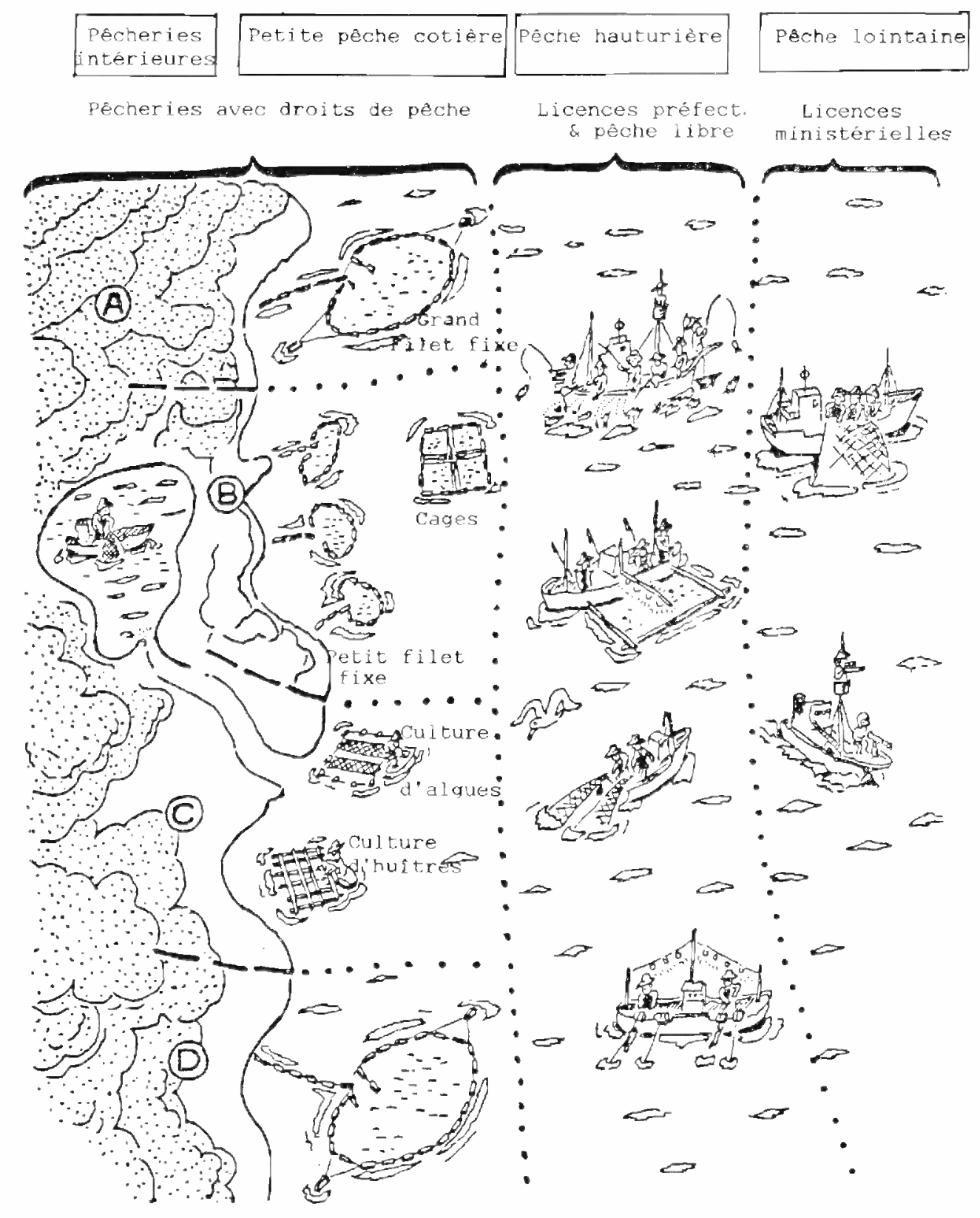

(A) (B) (C) (D) Coopératives de Pêcheurs

Fı. 2. - Systeme d'aménagement des pêches. 
pêche et des cultures marines (fig. 2). Ce schéma est bacé sur derrx conceptions: l'attribution des espaces (droits d'usage) et la limitation des capacités de capture (licences). Il est administré essentiellement au nivean lncal pour ce qui est des pêches cotières et de l'aquaculture.

\section{A) DROITS D'USAGE.}

Les droits d'usage territoriaux consistent en une attribution d'espaces dont l'usage est exclusivement réservé aux détenteurs de ces droits. II cxiste trois catégories de droits d'usage : les droits communautaircs, les droits territoriaux et les droits d'utilisation de filets fixes. Ils s'appli quent à des activités dont il est facile de délimiter le rayon d'action.

Les droits communautaines sont attribués poux des espaces où se pratiquent des pêcheries visant des espèces fixes ou sédentaires (algues, crustacés, poissons de fond) et des activités utilisant des engins fixes (casiers) ou relativement immobiles (seines de plage). Ces droits concernent la bande côtière adjacente au territoire des communes où il existe une coopérative de pêcheurs. La largeur de cette bande est variable. Elle est en moyenne de un kilomètre.

Le droit de pêche communautaire est attribué à la coopérative. Il s'agit d'un droit de propriété équivalent à un titre de propriété foncière. Il peut faire l'objet d'hypothèques ou de vente. Si l'Etat veut reprendre ce droit il doìt lc racheter à la coopérative. Seule la location est en droit prohibée. Le bénéfice de l'usage est exclusivement réservé aux membres de la coopérative. Celle-ci est responsable de l'administration de ce droit (utilisation de l'espace et exploitation des ressources). Les décisions en la matière sont prises à la majorité des deux ticrs de l'assemblée générale. C'est ainsi que l'allocation des sites peut faire l'objet de réglementations (nombre d'utilisateurs, fermeture,..). Pratiquement la quasi-totalité du littoral japonais est ainsi placé sous juridiction.

Les droits territoriaux concernent l'aquaculture. Ils sont en général attribués aux coopératives. Celles-ci sont chargées de l'allocation des sites aux membres qui désirent développer ce type d'activité. Afin d'assurer une certaine égalité entre les utilisateurs, des systèmes de rotation sont couramment pratiqués. Le caractère mobile de certaines structures de production facilite ces actions. Dans le cas de grands projets nécessitant la délimitation d'espace par des ouvrages importants, ces droits peuvent être attribués à des entreprises privées.

Les droits d'utilisation de filets fixes s'appliquent aux filets de grande taille fixés à des profondeurs supérieures à 27 mètres. Ces filets sont généralement localisés en dehors de la zone couverte par les droits communautaires. Les emplacements sont réalloués chaque année par la coopérative par tirage an sort.

\section{B) LICENCES.}

Les licences visent à un contrôle de l'effort de pêche total. Elles s'appliquent aux pêches de capture d'espèces mobiles. Elles sont une autorisation de construction d'une unité de pêche (bateaul) d'une certaine 
catégorie. Elles peuvent être assorties de limitations techniques quant au type de pêche pratiqué et, éventuellement, de quotas de capture. C'est le cas des pêcheries soumises à des quotas résultant de négociations internationales. C'est en fait un "droit à investir ". Le nombre de ces licences est déterminé par l'autorité administrative responsable de leur délivrance.

Dans le cas des pêcheries dites "protégées " (13 pécheries de grande pêche ou faisant l'objet d'accord internationaux) les licences sont délivrées par le Ministère de l'Agriculture, des Forêts et des Pêches. Les bateaux dont le rayon d'action ne dépasse pas l'extension en mer des limites terrestres des préfectures reçoivent des licences préfectorales. Dans le cas d'une pêcheric exploitant plusieurs zones préfectorales, les licences sont attribuées par le préfet de région dans les limites d'allocations fixes déterminées par le ministère.

Dans tous les cas, la méthode de calcul du nombre de licences et les procédures de demande et d'octroi sont semblables. Ce nombre est fixé en vue des résultats physiques (production) et comptables (rentabilité) des unités en activité. Ces caractéristiques d'exploitation permettent de définir un quota global garant du maintien de la productivité des stocks et d'un niveau moyen de rentabilité acceptable. Sur cette base on calcule le nombre potentiel de navires en fonction de leurs caractéristiques. ILes licences sont attribuées pour chaque pêcheric par classe de tonnage (principal critère retenu pour la mesure de l'effort), zone el saison de pêche. Elles sont délivrées nominativement au pêcheur pour son bateau, ou à l'entreprise poux chacun de ses navires. Elles peuvent éventuellement être assorties de limitations techniques concernant la puissance du moteur, les caractéristiques des engins, les conditions d'utilisation,... etc.

Ces licences ne peuvent faire l'objet de transactions commerciales directes. Elles doivent être, en théorie, remises "au pot commun ", pour être réallouées. Si le nombre global de licences est maintenu constant, toute nouvelle unité devra bénéficier d'un transfert de licence d'une unité quittant la pêcherie. Si la réduction de l'effort total est jugée nécessaire une procédure de retrait peut être initiée. Les navires perdant leur licence reçoivent une compensation financée par une contribution des bateaux restants et une aide de l'Etat.

\section{C) ADMINISTRATION DU SYSTEME DE GESTION DES PECHES.}

On a vu précédemment que les coopératives de pêcheurs jouent un rôle essentiel dans la gestion des droits d'usage. L'organigramme du systeme d'attribution des droits ainsi que des licences repose sur des commissions d'harmonisation (cu de coordination). Dans chaque préfecture, et au niveau national, de telles commissions sont constituées. Elles sont composées de représentants des pouvairs publics, des professionnels et de scientifiques. Juridiquement elles n'ont qu'une fonction de proposition et d'avis. De fait ceux-ci sont généralement suivis. Elle a un rôle d'arbitrage dans les conflits qui ne peuvent être résolus au sein des coopératives cu entre les coopératives.

La gestion d'ensemble s'appuie sur un réseau statistique performant en matière de débarquements et de comptabilité des entreprises. 


\section{UN PEU D'HISTOIRE.}

On peut présenter en quelques mots les grandes étapes qui ont conduit an systeme que l'on connait actuellement.

Le schéma d'aménagement des pêches résulte d'une adaptation progressive des droits et pratiques coutumiers instaurés à l'éponue féodale Les pratiques sont souvent le produit de plusieurs siecles d'observation. 1.a protection du droit de pêche au bénéfice exclusif des communautés de pêcheurs répondait à une avalanche de conflits soumis à l'arbitrage seigneural. Ils étaient aussi un moyen daffirmer l'autorité des propriétaires de grands filets (filets fixes et seines de plage), les plaçant ainsi à un rang proche de celui des propriétaires terriens dans l'ordre féoda!.

En 1868 la restauration, ou révolution, de Meiji remet l'administration de ces droits entre les mains du gouvernement central. Celui-ci maintient l'ensemble des droits jusqu'en 1875. Cette date correspond à une période de grande effervescence législative. Les juristes, de retour de voyages d'étude en Europe, ont des idćes bien précises sur les bases légales à donner à leur nouveau régime. Les notions de libre-accès et de prééminence de l'Etat sur le domaine maritime sont diffusées et en 1875 le gouvernement décide de nationaliser tout le littoral marin, annulant ainsi les droits traditionnels. L'expansionnisme inévitable de certains groupes et communautés conduit en quelques années à des situations inextricables de conflits et de confusion. Le gouvernement doit revenir sur sa décision en 1886 et rétablir l'ensemble des droits. Chaque préfecture est chargée de rédiger un règlement des pêches codifiant l'ensemble des droits et pratiques locales. La figure 3, extraite d'un document de 1898, reprend les repères permettant de définir un site attribué à une pêcherie au filet fixe. Ce site est encore exploité aujourd'hui. L'augmentation de la taille des filets a seulement nécessité un déplacement de l'engin vers le large.

\section{A) DEBUT DU SIECLE : LE BESOIN D'UNE COHERENGE D'ENSEMBLE.}

Le besoin d'un cadre juridique global pour la gestion de ces règlements locaux suscite l'élaboration d'une loi des pêches en 1901 (loi de Meiji). Celle-ci inclut les droits territoriaux, les principes de réglementation des activités cotières et les conditions de formation et de représentativité d'associations de pêcheurs. Par une réforme de cette loi en 1910 les droits de pêche ainsi que les droits d'accès (première forme des droits communautaires) deviennent des titres de propriété auxquels s'appliquent mutatis mutandis les attributions des droits de propriété terrestres.

La période suivante voit le développement de la motorisation et une extension progressive des pêches vers le large. Le rôle leader des activités de culture traditionnelles et des pêcheries fixes disparaît au profit de la pêche embarquée. De nombreux conflits d'intérêt, de sites,... en sont la conséquence. L'accroissement des apports, la généralisation des transactions commerciales et le développement des marchés urbains donnent de plus en plus de place aux intermédiaires. Ceux-ci confisquent la plus 




Fig. 3. - Schéma de localisution d'un sire réservé pour la pêche au filel fixe (1898).

grande part de la valeur ajoutée et de véritables révoltes de pêcheurs éclatent. La montée du militarisme, puis la guerre, empêcheront que ne soient apportées des solutions satisfaisantes à ces conflits.

B) APRES-GUERRE : UNE REGLEMENTATION PLUS COMPLETE POUR UN SECTEUR PLUS PERFORMANT.

Ceci ne sera fait qu'avec les deux lois, la Loi des Pêches adoptée en 1949, et la Loi sur les Associations Coopératives de Pêcheurs de 1951, encore en vigueur actuellement. La première reprend les droíts d'usage et institutionnalise le système des licences, licences qui existaient déjà pour certaines activités. Les soucis majeurs affichés dans cette réglementation sont l'augmentation de la productivité, l'amélioration du statut économique et social des pêcheurs et la "démocratisation " (littéralement traduit du japonais) de leurs associations. C'est dans ce cadre que les pêches se sont rapidement développées " de la côte vers la haute mer et vers le grand large ». Durant les années 1970 le revenu des pêcheurs a dépassé le revenu moyen national. Les cultures marines ont bénéficié d'une forte avancée de la connaissance biologique et du développement des techniques dans un environnement physique très favorable. 
L'autorité des conpératives de pêcheurs a été progressivement renforcée. Responsables de l'administration des droits de pêche, elles se sont vues confier l'exclusivité de la commercialisation, la responsabilité de la diffusion des techniques, le monopole du crédit à taux bonifić et enfin le rôle de porte-parole des intérêts des pêcheurs dans toms les conflits qui ont surgi depuis. Hormis les conflits internes aux péches, ceux ci ont princi palement opposé les pêcheurs aux industries et aux villes. T.es années du miracle économique, décennies soixante et soixante-dix, ont sacrifié une grande part de la qualité de l'enviromement à la croissance. I es pêcheurs en ont été souvent les premiers pénalisés.

Dans une période plus récente l'augmentation des coûts énergétiques et l'extension des zones de 200 milles ont contraint les Japonais à revenir dans leurs eaux. Le retour du grand large vers la côte a posé de nombreux problèmes de restructuration. Aujourd'hui ce sont les activités touristiques qui soumettent le schéma de l'aménagement à une nouvelle épreuve.

\section{III. - ORIGINALITE, DIFFICULTES ET PERSPECTIVES.}

Le système japonais d'aménagement des pêches présente une continuité des conceptions, des pratiques et des droits qui y sont associés que l'on ne retrouve que de manière anccdotique dans les autres pays industriels. Il reflète pour une bonne part les caractéristiques culturelles et les conditions économiques et sociales du développement du Japon. Mais sa souplesse et sa relative efficacité justifient que l'on s'arrête un instant sur les principes et les mécanismes qui en font l'originalité, ainsi que ses limites. On a retenu ici cinq aspects qui tranchent avec les conceptions généralement admises en matière de pêche et deux autres qui en tracent les limites et les perspectives.

\section{A) LA NOTION D'EXCLUSIVITE.}

Juridiquement l'exploitation des ressources halieutiques est prohibée excepté pour la petite pêche à la ligne en dehors des eaux communautaires. Elle doit faire l'objet d'une autorisation spéciale. Celle-ci est exclusive. Elle peut être attribuée directement aux bénéficiaires (licences, droit d'usage délivrés à des entreprises privées) ou indirectement par l'intermédiaire des organisations professionnelles. Ce principe est en opposition totale à celui du libre-accès.

\section{B) LE DESENGAGEMENT DE L'ETAT.}

L'autorité de l'Etat ne se trouve pas engagée dans l'administration des droits et des licences réglementant les activités dont le rayon d'action est limité localement. Dans la mesure du possible l'administration et l'arbitrage sont laissés aux mains des organisations professionnelles et des autorités locales. L'Etat se réserve le droit d’intervenir pour des raisons d'intérêt public, tout comme il le fait en matière terrestre. 
Les droits communautaires, droits de propriété détenus par les coopératives, ne constituent pas une réelle privatisation du domaine marilime. Mais en réservant de façon permanente les droits d'accès, d'usage et de modification de la configuration de l'espace à un groupe social, ils sont en contradiction avec la notion d'éminence du domaine de l'Etat qui s'applique au domaine maritime en France.

\section{C) VALEUIR FONCIERE DES ESPACES MARINS.}

Aux droits d'usage est attachée la notion de valeur qui caractérise un titre de propriété. Dans le cas de l'étroite bande administrée par les coopératives ce droit est permanent. Toute action visant ì une utilisation de l'espace ou de ses ressources à des fins autres que les activités des membres de la coopérative, doit faire l'objet d'un accord préalable de celle-ci. Si elle le juge nécessaire elle peut exiger une compensation. Dans le cas où cette action résulte en une perte de surface, le titre de propriété attaché à cet espace doit être racheté. Le prix d'achat est négocié par la coopérative et aucune action ne peut être entreprise avant que la transaction ne soit conclue. C'est ainsi que pour la création de la plus importante aire de stockage d'hydrocarbures du Japon, le gouvernement et les compagnies pétrolières ont dû racheter 62 hectares de mer aux coopératives de la baie de Shibushi (île de Kyushu) pour la somme de 250 millions de yens (10 millions de francs). Ce montant a été versé au terme d'un accord mettant fin à une opposition de 15 années des populations riveraines, et principalement des pêcheurs.

\section{D) LA SANTE ECONOMIOUE DES ENTREPRISES ET DU SECTEUR.}

On vient de voir trois notions, exclusivité, désengagement de l'Etat et valeur foncière, qui ne sont pas, ou mal, admises en matière de Domaine Public Maritime en France. Le critère de santé économique des entreprises et du secteur n'est pas en soi une spécificité japonaise. L'orjginalité se situe en cela qu'il constitue un des critères de base de la prise de décision. C'est la rentabilité des unités qui justifie le système des licences. Les limites ainsi imposées à la competition paraissent aujourd'hui une saine mesure. Mais pour se préoccuper des conséquences du surinvestissement sur les résultats des entreprises, les Japonais n'ont pas eu à forcer la main au principe du libre acces. L'histoire des conflits pour l'utilisation du littoral leur avait donné des leçons qu'ils n'ont pas oubliées.

Ce souci de préserver les performances économiques des entreprises transparaît à tous les niveaux de la réglementation et des processus de prise de décision. L'article premier de la loi sur les Associations Coopératives de Pêcheurs stipule comme objectif celui de "faire contribuer les pêcheurs et l'industrie de la transformation à l'économie nationale par l'augmentation de leur prođuctivité et l'amélioration de lcur statut économique et social ".

\section{E) LA RESPONSABILITE DE LA PROFESSION}

Les performances du système coopératif japonais font l'admiration des 
pêcheurs occidentaux. Celles-ci, rélles, Irouvent leurs londements dans la continuté de l'organisation sociale qu'elles incament ct dans le caractère légal des attributions des coopératives. Ies communautés de pêcheurs ont toujours été fortement structurées et représentées à partir du moment ou les diffecents pouvoirs qui se sont succédé ont è à arbitrer une multitude de conflits.

L'appartenance aux coopératives, qu'clles soient locales, de métier, ou autres, est obligatoire. C'est au sein de ces coopératives que se jouent toutes les négociations en matière de gestion des sites et dattribution de licences. Filles ont un poids considérable sur les décisions de l'administration. Elles sont parfois critiquées pour leur trop grande rinidité, mais elles restent 11 des piliers de l'aménagement des pêches. Leur ròle dans la commercialisation et la valorisation est aussi un élément important de la vitalité du secteur.

\section{F) LIMITES ET PERSPECTIVES.}

Ainsi présentées, les pêcheries japonaises peuvent sembler à l'abri des difficultés que nous conmaissons. En fait elles offrent l'image d'un dynamisme d'ensemble qui cache des conflits parfois très durs. On les retrouve bien évidemment au niveau des intérêts locaux dans les coopératives. Mais ils existent aussi à une échelle plus large sans que puissent être données des réponses d'ensemble satisfaisantes. On se limitera ici à la généralisation des pratiques de revente des droits communautaires et au découpage excessif de la gestion du littoral. Mais on pourrait aussi aborder les difficultés liées aux performances d'activités nouvelles très rémunératrices qui convoitent les sites d'activités anciennes moins rentables.

\section{G) " VENDRE SA MER? "}

L'espace littoral est l'enjeu d'une compétition acharnée depuis les années 1960. Les arguments financiers des villes et de l'industrie ont séduit de nombreuses coopératives. Le lassement du revenu moyen des pêcheurs, la difficulté des coopératives à honorer leurs engagements financiers dans des grands projets d'aménagement, suscitent actuellement une recrudescence des pratiques de vente des droits communautaires. Elles posent un grave problème aux pêcheurs à la fois en termes d'accès, mais aussi de préservation des équilibres écologiques. Les surfaces rachetées sont généralement comblées ce qui entraine la destruction de frayères, la modification de la courantologie,... De nombreux pêcheurs ont ainsi définitivement perdu des fonds très productifs, pertes que les indemnisations ne compensent que temporairement. La conséquence logique est l'arrêt, immédiatement ou à moyen terme, d'une partie des activités de pêche.

\section{H) L'AMENAGEMENT INTEGRE.}

Le développement de l'aquaculture et les perspectives offertes par l'évolution des techniques d'élevage ot de manjpulation des écosystèmes, posent de graves problèmes d'aménagement et de gestion de l'espace 


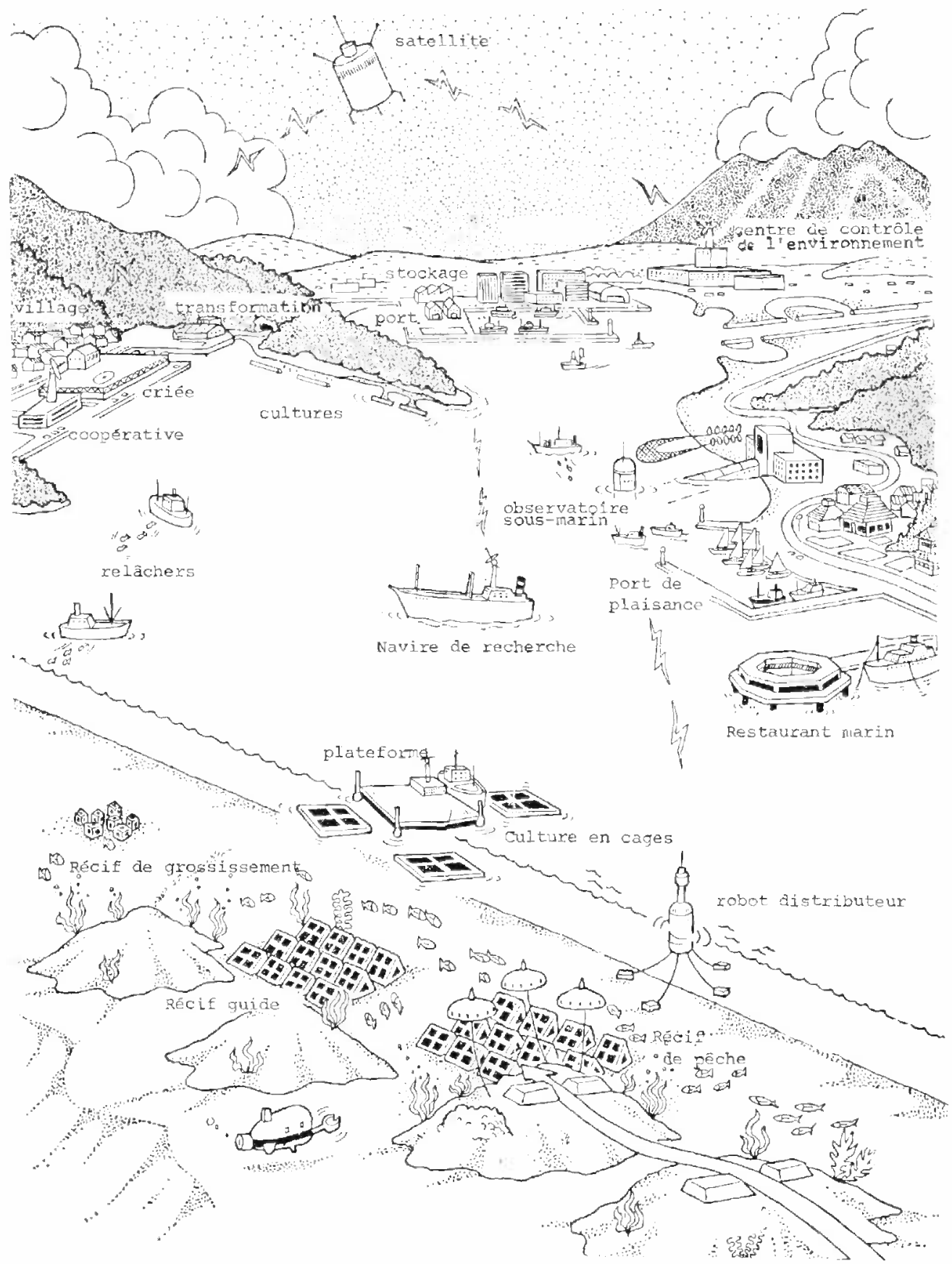

F1G. 4. - Schéma d'utilisation de l'espace du type "marinovation ». 
littoral marin. Dans les zones les plus propices à l'aquaculture on constate des problèmes de surcharge. Ils affectent les productions mais aussi la qualité de l'environnement. La diffusion des nuisances, le developpement de l'aquaculture vers le large, la gestion de programmes de repeuplement, sont quelques-unes des questions qui se heurtent à la rigidité d'une administration excessivement découpée de la bande littorale.

Une gestion ne pourra être effective, et utiliser au mienx les opportnnités offertes par les techniques nouvelles, que si elle est intégree à une échelle plus large que celle des coopératives locales. Le cadre préfectoral est trop large pour permettre des interventions décisives dans l'ensemble de ces domaines. Il est nécessaire de trouver une unité de gestion intermédiaire, qui se situe au niveau des entités biologiques, écologiques et économiques que sont les bassins, lorsque leur gestion dépend de l'autorité de plusieurs coopératives.

C'est pour tenter de répondre à ce besoin que les projets du type "Marinovation" se multiplient actuellement. La figure 4 est une vision globale de l'aménagement de la baie de Kagoshima. Au-delà des aspects futuriste et simpliste de la représentation on y voit transparaître le souci d'une gestion scientifique et d'une intégration des activités touristiques. Celles-ci, jusqu'alors peu consommatrices de littoral, se développent très rapidement avec de gros moyens financiers.

La réalisation effective des projets "Marinovation ", et autres, est en partie tributaire d'une réforme des cadres institutionnels de l'aménagement et de ses conceptions fondamentales.

\section{Conclusion.}

En guise de conclusion il est peut-être nécessaire de dire que nous analysons et comprenons un schéma d'aménagement comme un ensemble de mécanismes sociaux, économiques et juridiques d'allocation de l'espace et des ressources à des usages concurrents. En pêches ces mécanismes participent du processus de réalisation et de partage des bénéfices réalisés au cours du processus d'exploitation des ressources halieutiques. Il n'existe pas d'aménagement en soi, mais seulement des objectifs et des choix qui s'intègrent dans le contexte historique, économique, social et politique, d'une société. Il n'y a donc pas de "modèle ", mais des valeurs, des conceptions et des hiérarchisations qui doivent être jugées à l'aune du contexte où elles s'appliquent. Celles-ci peuvent servir à éclairer les choix à effectuer dans d'autres conditions.

C'était l'objectif de cet exposé qui ne cherchait pas à nourrir une comparaison, ni à imposer une vision de l'aménagement qui a montré ses limites. Ceci d'autant plus que si les Japonais jouissent d'une avance confortable en matière de gestion des pêches ils sont toujours à la recherche d'une loi littorale. 
